\title{
Adaptive control for optimizing microalgae production
}

\author{
Francis Mairet* Rafael Muñoz-Tamayo* Olivier Bernard ${ }^{*, * *}$ \\ * BIOCORE-INRIA, BP93, 06902 Sophia-Antipolis Cedex, France \\ (e-mail: \{francis.mairet, rafael.munoz_tamayo, \\ olivier.bernard\}@inria.fr) \\ ** LOV-UPMC-CNRS, UMR 7093, Station Zoologique, B.P. 28 06234, \\ Villefranche-sur-mer, France
}

\begin{abstract}
In this paper, we propose a nonlinear adaptive controller for light-limited microalgae culture. This controller regulates the light absorption factor, defined by the ratio between the incident light and the light at the bottom of the reactor. Then, we propose a set-point for the light absorption factor which allows to optimize biomass productivity under constant illumination. Finally, we show by numerical simulation that the adaptive controller can be used to obtain near optimal productivity under day-night cycles.
\end{abstract}

Keywords: Nonlinear controller, Microalgae, Optimization, Biomass production, Light-limited culture, Luminostat.

\section{INTRODUCTION}

Recently, microalgae cultivation has become more and more popular for the mass production in the industry of food, aquaculture, pharmaceutics and energy (Spolaore et al., 2006; Chisti, 2007; Wijffels and Barbosa, 2010). However, this promising domain is still young, and there is still a large progression margin to improve economic and environmental yields for large scale productions.

In this context, online control of microalgae culture in order to increase productivity is becoming a key research topic (Berenguel et al., 2004; Akhmetzhanov et al., 2010; Bernard, 2011; Ifrim et al., 2013; Tebbani et al., 2013). The periodic forcing of outdoor microalgae culture, due to the day-night cycles, is a specificity of this domain which makes control and optimization more challenging.

Here, our objective is to propose a closed loop control which allows near optimal biomass productivity for lightlimited culture (assuming that all nutrients are supplemented in excess). For this end, we design an adaptive controller which regulates the light absorption factor, defined by the ratio between the incident light and the light at the bottom of the reactor. Originally developed for constant light conditions (Mairet et al., 2013), the controller is extended to time-varying illumination. The structure of the controller, based on the work of Mailleret et al. (2004), is of particular interest for bioprocesses since it does not require any knowledge of the growth rate kinetics. Then, we will show that this controller can be exploited to optimize microalgae production.

The paper is organized as follows. We present firstly a model for light limited culture (Huisman and Weissing, 1994). This framework will be the basis of our develop- ment. In Section 3, we present an adaptive controller which regulates the light absorption factor. Then, we show that this controller can be used in order to optimize biomass production in light/dark cycles. Finally, we illustrate our approach with numerical simulations: the adaptive controller allows obtaining productivity close to the optimum (determined numerically).

\section{MODELLING LIGHT-LIMITED GROWTH OF MICROALGAE}

This section presents a simple model for light-limited growth of microalgae, based on the research work developed by Jef Huisman and co-workers (Huisman and Weissing, 1994; Weissing and Huisman, 1994; Gerla et al., 2011) for competition. Originally developed for natural systems (lake, ocean...), this theoretical approach can be adapted and exploited in the framework of microalgae culture systems (planar photobioreactor or raceways).

\subsection{Model development}

Let us consider a mixed microalgae culturing system of depth $\mathrm{L}$ in which grows a biomass $x$ of microalgae. We assume that the system is completely homogeneous, i.e., the concentration of microalgae is the same in all the points of the reactor. Due to light absorption and diffusion by the algae, a spatial gradient of light occurs: the light intensity in the reactor decreases along the depth.

Let us assume that the absorption of light in the water column follows the Lambert-Beer law. Thus, for a given depth $z$, the corresponding light intensity $I(x, z, t)$ satisfies

$$
I(x, z, t)=I_{\text {in }}(t) \exp (-a x z)
$$


where $I_{i n}(t)$ is the incident light, and $a$ is the coefficient of attenuation due to microalgae. The light at the bottom of the water column is called $I_{\text {out }}(x, t)=I(x, L, t)$.

We consider here that the growth of microalgae is only limited by light, and it is a continuous function defined by the specific growth rate $\mu(I)>0, \forall I>0$. The total growth $G$ of microalgae is obtained by integrating the local growth over depth:

$$
G(x, t)=\frac{x}{L} \int_{0}^{L} \mu(I(x, z, t)) d z
$$

Given Equation (1), we obtain

$$
G(x, t)=\frac{1}{a L} \int_{I_{\text {out }}(x, t)}^{I_{\text {in }}(t)} f(I) d I
$$

with $f(I)=\mu(I) / I$.

Given a constant dilution rate $u^{1}$, the dynamic evolution of the microalgae concentration $x$ is therefore given by

$$
\dot{x}=G(x, t)-u x .
$$

\subsection{Model analysis}

In this subsection, as in Mairet et al. (2013), we consider a constant light supply $I_{i n}$. First, note that $G(x)$ is an increasing function whose derivatives write:

$$
\begin{aligned}
& G^{\prime}(x)=\mu\left(I_{\text {out }}(x)\right)>0 \\
& G^{\prime \prime}(x)=-a L \mu^{\prime}\left(I_{\text {out }}(x)\right)
\end{aligned}
$$

Thus, the asymptotic behavior of the model depends on the growth rate:

i. For an increasing growth rate $\mu(I)$ (Huisman and Weissing, 1994; Weissing and Huisman, 1994)

Proposition 1. For increasing growth rate, if $u<\mu\left(I_{i n}\right)$, then Equation (4) has one non-trivial equilibrium, which is globally stable.

Proof. For increasing growth rate, $G(x)$ is increasing and concave given Equation (5) (see Figure 1). Thus, System (4) is bounded and has two equilibria if $u<\mu\left(I_{i n}\right)=$ $G^{\prime}(0)$ :

- a trivial equilibrium which is unstable,

- a non-trivial stable equilibrium.

For example, assuming a kinetics of Michaelis-Menten type, the growth rate writes:

$$
\mu(I)=\bar{\mu} \frac{I}{I+K_{s}} .
$$

The total growth $G$ is obtained by integration along the depth:

\footnotetext{
1 Initially, Huisman and co-workers used a loss rate which gathers dilution, mortality, predation... In a microalgae production system, we first assume that the loss is mainly due to dilution.
}
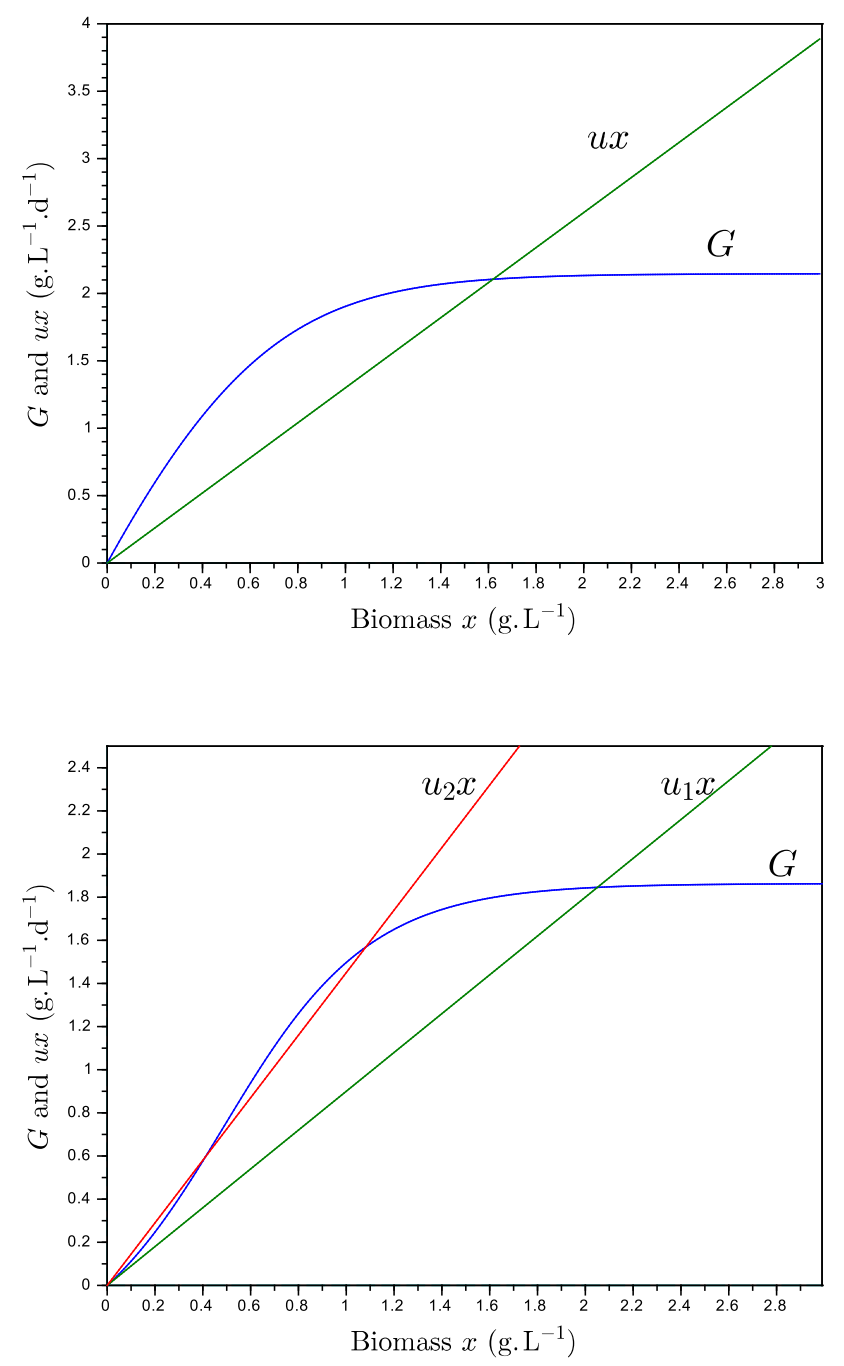

Fig. 1. Growth $G(x)$ and dilution $u x$ as a function of biomass $x$. Top: for Monod growth rate (one stable equilibrium). Bottom: for Haldane growth rate (one or two equilibria). See Section 2.2.

$$
G(x)=\frac{\bar{\mu}}{a L} \ln \left(\frac{I_{\text {in }}+K_{s}}{I_{\text {out }}(x)+K_{s}}\right) .
$$

The nontrivial equilibrium of (4) is obtained by solving

$$
\frac{\bar{\mu}}{a L} \ln \left(\frac{I_{i n}+K_{s}}{I_{i n} \exp \left(-a x^{\star} L\right)+K_{s}}\right)=u x^{\star},
$$

where $x^{\star}$ is the biomass concentration at steady state. This allows to define the light at the bottom of the water column at equilibrium $I_{\text {out }}^{\star}=I_{\text {in }} \exp \left(-a x^{\star} L\right)$.

ii. For a non-monotone growth rate $\mu(I)$ (photoinhibition) We now consider the case where the growth function has one maximum. For example, the specific growth rate can be represented by a Haldane function :

$$
\mu(I)=\bar{\mu} \frac{I}{K_{s I}+I+I^{2} / K_{i I}},
$$


In this case, the total growth $G$ obtained by integration over depth writes, considering that $K_{i I}<4 K_{s I}$ (Bernard et al., 2009; Bernard, 2011):

$$
\begin{gathered}
G(x)=\bar{\mu} \frac{2 K_{i I}}{a L \sqrt{\Delta}}\left[\arctan \left(\frac{2 I_{\text {out }}(x)+K_{i I}}{\sqrt{\Delta}}\right)\right. \\
\left.-\arctan \left(\frac{2 I_{\text {in }}+K_{i I}}{\sqrt{\Delta}}\right)\right]
\end{gathered}
$$

where $\Delta=K_{i I}\left(4 K_{s I}-K_{i I}\right)$.

Proposition 2. For nonmonotone growth rate, Equation (4) can have one (globally stable) or two nontrivial equilibria. In this last case, one is locally stable and the other unstable, the trivial equilibrium is also locally stable.

Proof. Given Equation (5), $G(x)$ has an inflection point (see Figure 1) so the equation $G(x)=u x$ can have one or two non-trivial solutions. See Gerla et al. (2011) for more details.

This corresponds to a strong Allee effect: at low concentration, the specific growth rate increases with biomass concentration (self-shading reduces the negative impact of photoinhibition). Nevertheless, below a threshold biomass concentration (corresponding to the unstable equilibrium), the biomass goes extinct (Gerla et al., 2011). Operating a microalgae culture under high illumination in open-loop can lead to the wash-out of the biomass. Thus, the design of closed-loop control strategies for light-limited culture is a necessity for the development of large-scale biomass production.

\section{ADAPTIVE CONTROLLER}

Now, we propose to use the dilution rate $u$ as a control in order to regulate the light attenuation factor $y=a x$ to a desired set-point $y^{*}$ in closed loop.

\subsection{Controller design}

In the following, we assume the availability of two measurements:

Hypothesis 3. We consider that the following measurements are available:

- the light attenuation factor:

- the total growth $G$

$$
y=a x=\frac{1}{L} \ln \left(\frac{I_{\text {in }}}{I_{\text {out }}(x)}\right)
$$

The light attenuation factor $y$ can be easily computed from light measurements by online sensors at two places in the reactor. The total growth $G$ can be estimated using observer-based estimator (Bastin and Dochain, 1990; Perrier et al., 2000; Mairet et al., 2010) based on measurement of oxygen production (Mendoza et al., 2013) or $\mathrm{CO}_{2}$ consumption for example.

We propose a feedback law (based on the work of Mailleret et al. (2004); Mairet et al. (2013)) which involves an adaptive gain $\gamma(t)$. From $y^{*}$, one can define the corresponding set-point $\gamma^{*}=\frac{a}{y^{*}}$.

Theorem 4. Consider an incident light $I_{i n}(t)>\epsilon>$ $0, \forall t>0$. Under 3 , the adaptive feedback control law

$$
\left\{\begin{array}{l}
u(t)=\gamma(t) G(x, t) \\
\dot{\gamma}=K G(x, t)\left(y-y^{*}\right)\left(\gamma-\gamma_{m}\right)\left(\gamma_{M}-\gamma\right)
\end{array}\right.
$$

with $0<\gamma_{m}<\gamma^{*}<\gamma_{M}$ and $K>0$ globally stabilizes System 4 towards the positive set point $x^{*}=y^{*} / a$.

Proof. Given that $y=a x$, System (4) under control law (11) becomes:

$$
\left\{\begin{array}{l}
\dot{y}=G(x, t)(a-\gamma y) \\
\dot{\gamma}=K G(x, t)\left(y-y^{*}\right)\left(\gamma-\gamma_{m}\right)\left(\gamma_{M}-\gamma\right)
\end{array}\right.
$$

Since $G(x, t) \geq 0$, the set $\mathcal{R}^{+*} \times\left[\gamma_{m}, \gamma_{M}\right]$ is positively invariant (in the following, we assume initial conditions belonging to this set). Given its dynamics, one can show that $y(t) \geq \underline{y}, \forall t \geq 0$, with:

$$
\underline{y}=\min \left(y(0), \frac{a}{\gamma_{M}}\right) \text {. }
$$

So we have $I_{\text {out }}(t) \leq I_{\text {in }}(t) \exp (-\underline{y} L), \forall t \geq 0$. Using Equation (3), we deduce that $G(x, t)$ is lower bounded (since $f(I)>0, \forall I>0)$. Thus, we can introduce the time change $t^{\prime}=\int_{0}^{t} G(x(\tau), \tau) d \tau$. Denoting with a prime the derivatives with respect to $t^{\prime}$, System (12) rewrites:

$$
\left\{\begin{array}{l}
y^{\prime}=a-\gamma y \\
\gamma^{\prime}=K\left(y-y^{*}\right)\left(\gamma-\gamma_{m}\right)\left(\gamma_{M}-\gamma\right)
\end{array}\right.
$$

Now consider the following Lyapunov candidate function V (Mailleret et al. (2004)):

$$
V=\int_{y^{*}}^{y} \frac{w-y^{*}}{w} d w+\int_{\gamma^{*}}^{\gamma} \frac{w-\gamma^{*}}{K\left(w-\gamma_{m}\right)\left(\gamma_{M}-w\right)} d w
$$

The derivative of $V$ along the trajectories of System (13) is given by

$$
V^{\prime}=-a \frac{\left(y-y^{*}\right)^{2}}{y y^{*}}
$$

$V$ is a continuously differentiable, radially unbounded, positive definite function with $V^{\prime}(y, \gamma) \leq 0, \forall(y, \gamma) \in$ $\mathcal{R}^{+} \times\left[\gamma_{m}, \gamma_{M}\right]$. Moreover, one can easily check that the largest invariant set defined by $V^{\prime}=0$ is actually the set-point $\left(y^{*}, \gamma^{*}\right)$. Therefore, using Krasovskii theorem (Khalil, 2002), the set-point $\left(y^{*}, \gamma^{*}\right)$ is globally asymptotically stable.

Note that this control scheme does not require any knowledge of the growth rate or the attenuation coefficient. Moreover, the proposed controller can be used for multispecies culture: it will allow the selection of the fastest growing species under the condition fixed by the set-point (Mairet et al., 2013).

\subsection{Simulation}

Table 1. Parameter values used for simulation in Section 3.2.

\begin{tabular}{lc} 
Parameters & Values \\
\hline $\bar{\mu}$ & $5 \mathrm{~d}^{-1}$ \\
$K_{s I}$ & $300 \mu \mathrm{mol} . \mathrm{m}^{-2} \cdot s^{-1}$ \\
$K_{i I}$ & $400 \mu \mathrm{mol} . \mathrm{m}^{-2} \cdot s^{-1}$ \\
$a$ & $1 \mathrm{dm}^{2} \cdot g^{-1}$ \\
$L$ & $2 \mathrm{dm}$ \\
\hline
\end{tabular}



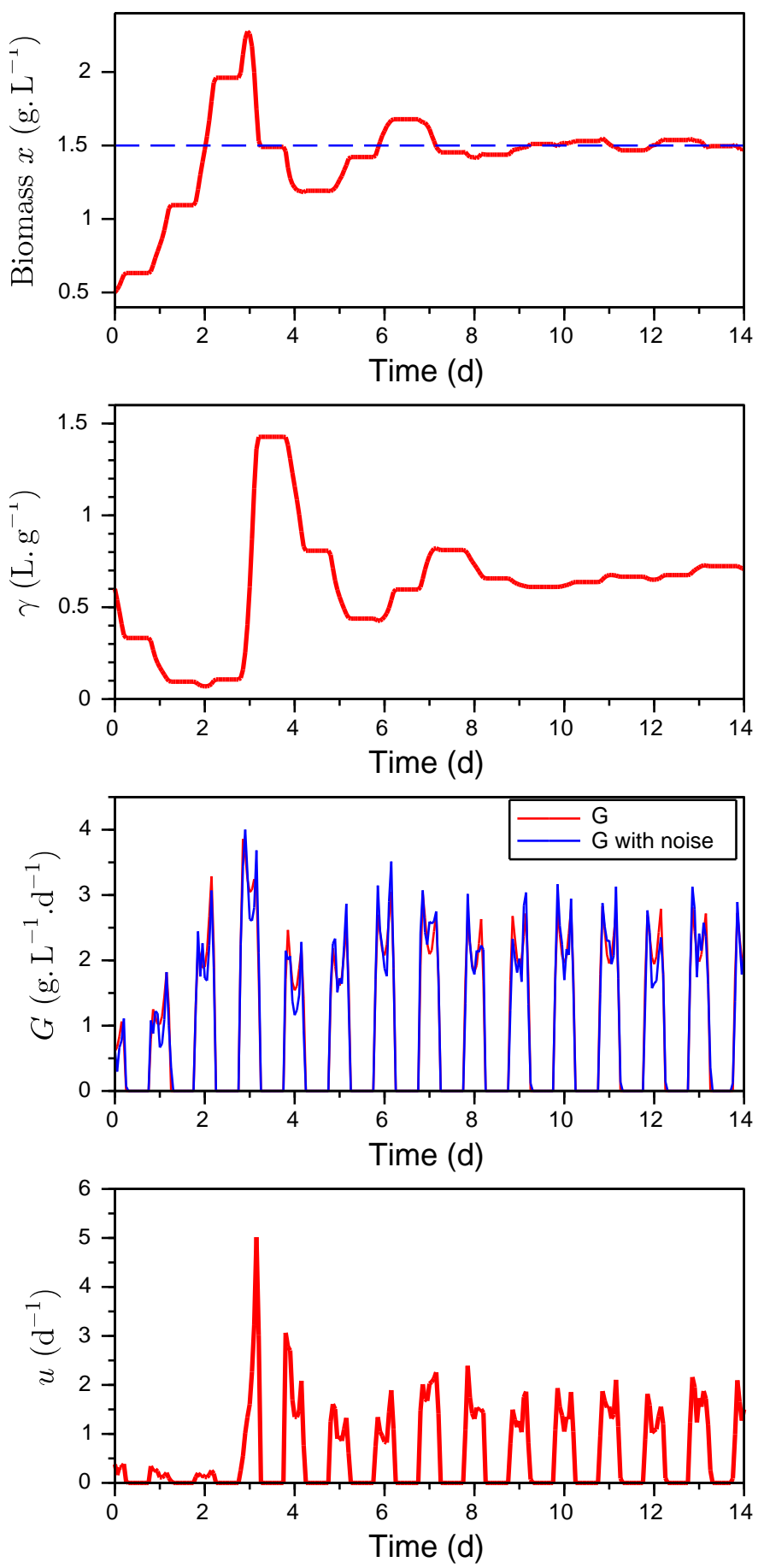

Fig. 2. Simulation of Model (4) with the Adaptive Control (11) under day-night cycles. The set-point is represented by the dashed line.

Model (4) with Haldane growth rate (Eq. (9)) under the Control law (11) is simulated with a periodic light supply:

$$
I_{i n}(t)=I_{o}[\max (0, \sin (2 \pi t))]^{2}
$$

where $I_{o}=1200 \mu \mathrm{mol} . \mathrm{m}^{-2} \cdot s^{-1}$. Growth measurements $G$ are corrupted by an additive noise (up to $0.5 \mathrm{~g} . \mathrm{L}^{-1} \cdot \mathrm{d}^{-1}$ ). Model parameters are given in Table 1. Tuning param- eters of the control law result from a trade-off between rapidity and smoothness: $K=1, \gamma_{m}=0.01 \mathrm{~L} \cdot \mathrm{g}^{-1}$, and $\gamma_{M}=4 \mathrm{~L} \cdot \mathrm{g}^{-1}$. The set-point is $y^{*}=1.5 \mathrm{dm}^{-1}$. Figure 2 shows the good performance of the controller, even in presence of a periodic forcing and noise measurements. Recall that the controller does not require any knowledge of model parameters. Note that we observe a decrease of growth $G$ at noon because of photoinhibition.

Nevertheless, in its present form, the controller requires the positiveness of $G$. This is no longer true in the night when respiration is considered. To overcome this problem, a practical implementation of the controller is proposed in the next section.

\section{OPTIMIZATION OF BIOMASS PRODUCTIVITY}

In this section, we will show that the adaptive controller (11) can be used in order to optimize biomass productivity $P=u x$. Respiration plays a major role in the definition of optimal conditions for biomass production. Thus, we add in the model a respiration rate $r$ that we first assume constant. $G$ is now defined as the net growth:

$$
G(x, t)=\frac{1}{a L} \int_{I_{\text {out }}(x, t)}^{I_{\text {in }}(t)} f(I) d I-r x
$$

Given that $G$ can be negative, the adaptive control law is modified as follows:

$$
\left\{\begin{array}{l}
u(t)=\gamma(t) \max (G(x, t), 0) \\
\dot{\gamma}=K \max (G(x, t), 0)\left(y-y^{*}\right)\left(\gamma-\gamma_{m}\right)\left(\gamma_{M}-\gamma\right)
\end{array}\right.
$$

Although convergence is no longer guaranteed, the system moves faster during the light phase (when $G(x, t)>0$ ) than the dark phase (when $G(x, t)<0$ ) so we can expect that the system converges towards a periodic solution around the set-point.

\subsection{Under constant light}

A criteria on the light at the bottom of the water column has been proposed in order to optimize biomass productivity (Takache et al., 2010):

Proposition 5. Under constant illumination, the steadystate biomass productivity is optimal when the light at the bottom of the culture $I_{\text {out }}^{*}$ is such that:

$$
\mu\left(I_{\text {out }}^{*}\right)=r .
$$

Proof. At steady-state, we have $P=u x=G(x)$. The maximum of $P$ is obtained for $G^{\prime}(x)=\mu\left(I_{\text {out }}(x)\right)-r=0$.

This operating mode is called luminostat.

Thus, under constant illumination, the adaptive controller (16) can be used to regulate the light attenuation factor at the set-point $y^{*}=\frac{1}{L} \ln \left(\frac{I_{\text {in }}}{I_{\text {out }}^{*}}\right)$ in order to optimize biomass productivity. 


\subsection{Under day-night cycle}

The optimization of biomass production under day-night cycle is more challenging. Indeed, the luminostat operation could not be maintained in this condition (Cuaresma et al., 2011). To tackle this problem, Muñoz-Tamayo et al. (2013) have determined numerically an optimal strategy. Then, following the approach of self-optimizing control, they have shown that the regulation of the light absorption allows to achieve near optimal productivity. Contrary to the optimal open-loop strategy, this closed-loop control is more robust towards environmental conditions and model uncertainties.

In the following, we will use our controller in order to regulate the light absorption and we will compare the productivity with the optimal trajectory.

\subsection{Simulation}

We test our approach in simulation with the model proposed in Muñoz-Tamayo et al. (2013). This model takes into account the effect of light, nitrogen and temperature. It represents the variation of biomass, nitrogen quota, chlorophyll, and lipid. Note that contrary to our simple model, the respiration rate is not constant and the light attenuation depends on the chlorophyll content of microalgae, which is affected by its nutrient status. The model was calibrated with experimental data of Isochrysis aff. galbana culture.

For a given time horizon $t_{f}$, the objective is to maximize biomass productivity:

$$
\begin{aligned}
& \max _{u(t)} \int_{t_{0}}^{t_{f}} u(t) x(t) d t . \\
& \text { s.t. } \\
& \quad 0 \leq u(t) \leq u_{\max } \\
& \quad \dot{\mathbf{x}}=\mathbf{g}\left(\mathbf{x}, f_{i}, t\right), \mathbf{x}(0)=\mathbf{x}_{0} .
\end{aligned}
$$

where $\dot{\mathbf{x}}=\mathbf{g}\left(\mathbf{x}, f_{i}, t\right)$ is the model proposed in MuñozTamayo et al. (2013) (Equations (1-5)).

The optimal solution is obtained numerically using the Matlab toolbox DOTcvpSB Hirmajer et al. (2009).

DOTcvpSB uses the approach of sequential discretization (control vector parameterization) to solve the non-linear programming (NLP) problem. The optimization was performed by using stochastic algorithms Storn and Price (1997); Runarsson and Yao (2000).

Then, we compare numerically the adaptive control (16) with the optimal solution (see Figure 3 ). First, note that the observed oscillations are both due to the forcing light signal and to the respiration during the night, when the controller set the system in batch mode. In particular, the light attenuation factor $y$ cannot tend to an equilibrium point, contrary to the previous simulation without respiration (see Figure 2). Although it has been designed on a simpler model, the adaptive controller presents good performance in terms of regulation. A periodic regime oscillating around the set-point is achieved after four days
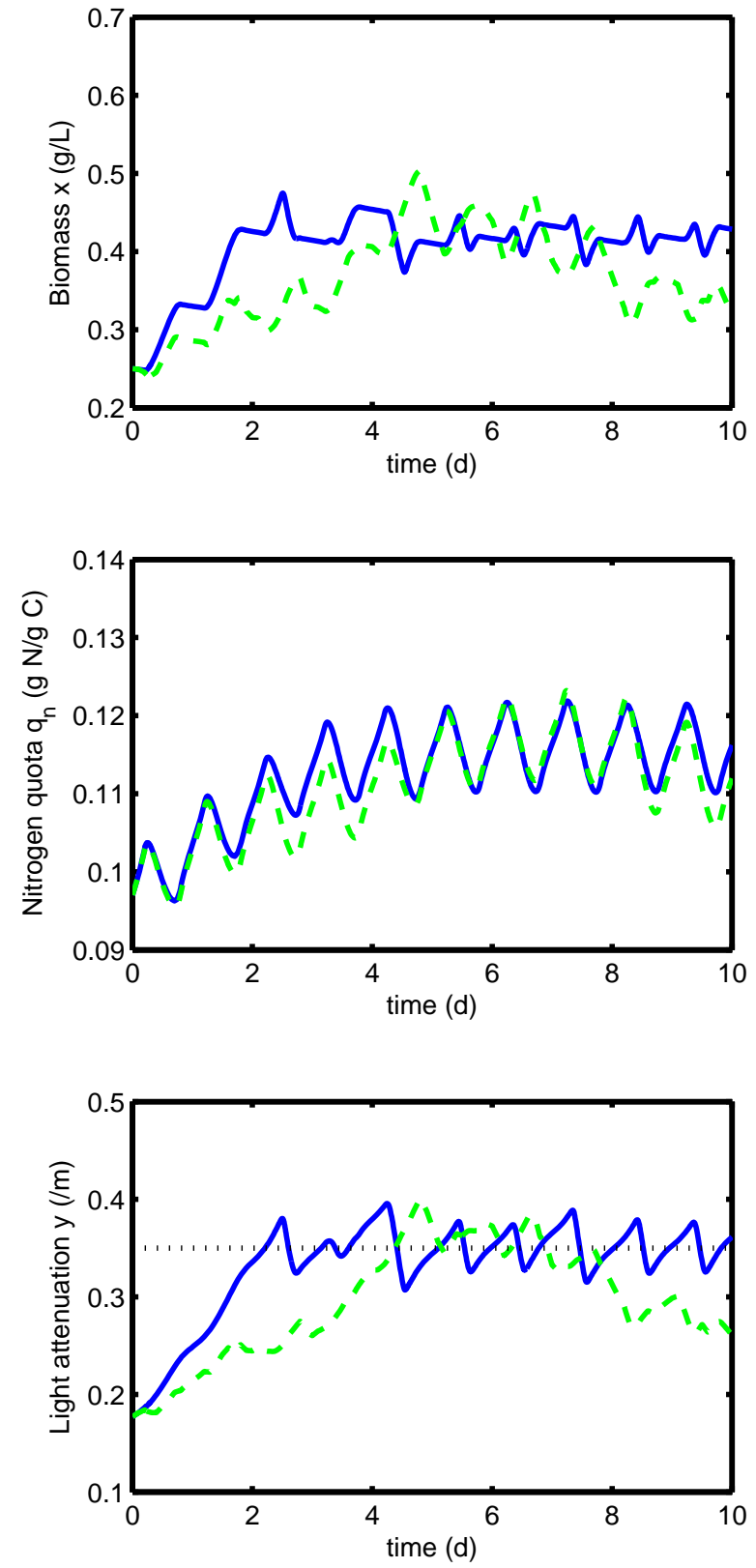

Fig. 3. Simulation with the model proposed in MuñozTamayo et al. (2013). Comparison between the optimal solution (green dashed line) and the adaptive controller (blue line). The set-point for the adaptive controller is represented by the dotted line.

of culture. Moreover, the controller obtains biomass productivity close to the optimal (see Figure 4). Nevertheless, the set-point $y^{*}$ for the adaptive controller has been chosen according to the numerical solution of the optimal problem. For real implementation, the set-point can be modified because of environmental conditions and model uncertainties. Such adaptive strategy will deserve further investigation.

\section{CONCLUSION}

In this paper, we have proposed a nonlinear adaptive control which regulates the light absorption factor in a 


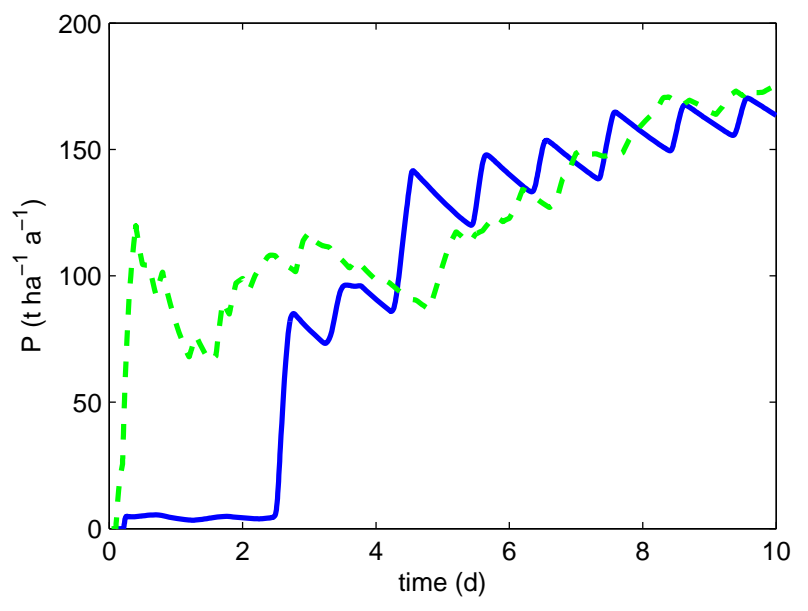

Fig. 4. Biomass Productivity. Comparison between the optimal solution (green dashed line) and the adaptive controller (blue line).

microalgae culture. The global asymptotic stability of the closed-loop system has been shown based on a simple model of light-limited growth. Then, it was shown through numerical simulations that the adaptive controller presents good performances in terms of regulation under constant light, and also in day-night cycle with a more realistic model including the effects of temperature and nitrogen quota. Such operation is of particular interest for optimizing biomass production. In the future, the performance of such controller will be tested experimentally.

\section{REFERENCES}

Akhmetzhanov, A., Grognard, F., Masci, P., and Bernard, O. (2010). Optimization of a photobioreactor biomass production using natural light. In Proceedings of the 49th CDC conference.

Bastin, G. and Dochain, D. (1990). On-line estimation and adaptive control of bioreactors. Elsevier, New York.

Berenguel, M., Rodriguez, F., Acien, F., and Garcia, J. (2004). Model predictive control of ph in tubular photobioreactors. Journal of Process Control, 14(4), $377-387$.

Bernard, O. (2011). Hurdles and challenges for modelling and control of microalgae for co2 mitigation and biofuel production. Journal of Process Control, 21, 1378-1389.

Bernard, O., Masci, P., and Sciandra, A. (2009). A photobioreactor model in nitrogen limited conditions. In Proceedings of the $6^{\text {th }}$ Conference on Mathematical Modelling. Vienna.

Chisti, Y. (2007). Biodiesel from microalgae. Biotechnology Advances, 25, 294-306.

Cuaresma, M., Janssen, M., Van den End, E.J., Vílchez, C., and Wijffels, R.H. (2011). Luminostat operation: A tool to maximize microalgae photosynthetic efficiency in photobioreactors during the daily light cycle? Bioresource technology, 102(17), 7871-7878.

Gerla, D., Mooij, W., and Huisman, J. (2011). Photoinhibition and the assembly of light-limited phytoplankton communities. Oikos, 120(3), 359-368.

Hirmajer, T., Balsa-Canto, E., and Banga, J. (2009). Dotcvpsb, a software toolbox for dynamic optimization in systems biology. BMC bioinformatics, 10(1), 199.
Huisman, J. and Weissing, F. (1994). Light-limited growth and competition for light in well-mixed aquatic environments: an elementary model. Ecology, 75, 507-520.

Ifrim, G.A., Titica, M., Barbu, M., Boillereaux, L., Cogne, G., Caraman, S., and Legrand, J. (2013). Multivariable feedback linearizing control of chlamydomonas reinhardtii photoautotrophic growth process in a torus photobioreactor. Chemical Engineering Journal, 218(0), 191 -203 .

Khalil, H. (2002). Nonlinear systems. Prentice Hall, third edition.

Mailleret, L., Bernard, O., and Steyer, J.P. (2004). Robust nonlinear adaptive control for bioreactors with unknown kinetics. Automatica, 40:8, 365-383.

Mairet, F., Moisan, M., and Bernard, O. (2010). Interval observer-based estimator of specific growth rate in bioreactors. Journal Européen des Systèmes Automatisés, 44(4-5), 493-507.

Mairet, F., Muñoz-Tamayo, R., and Bernard, O. (2013). Driving species competition in a light-limited chemostat. In 9th IFAC Symposium on Nonlinear Control Systems (NOLCOS).

Mendoza, J., Granados, M., de Godos, I., Acin, F., Molina, E., Heaven, S., and Banks, C. (2013). Oxygen transfer and evolution in microalgal culture in open raceways. Bioresource Technology, 137(0), 188 - 195.

Muñoz-Tamayo, R., Mairet, F., and Bernard, O. (2013). Optimizing microalgal production in raceway systems. Biotechnology progress, 29, 543-552.

Perrier, M., de Azevedo, S.F., Ferreira, E., and Dochain, D. (2000). Tuning of observer-based estimators: theory and application to the on-line estimation of kinetic parameters. Control Engineering Practice, 8(4), 377388.

Runarsson, T.P. and Yao, X. (2000). Stochastic ranking for constrained evolutionary optimization. Evolutionary Computation, IEEE Transactions on, 4(3), 284-294.

Spolaore, P., Joannis-Cassan, C., Duran, E., and Isambert, A. (2006). Commercial applications of microalgae. Journal of bioscience and bioengineering, 101(2), 87-96.

Storn, R. and Price, K. (1997). Differential evolutiona simple and efficient heuristic for global optimization over continuous spaces. Journal of global optimization, 11(4), 341-359.

Takache, H., Christophe, G., Cornet, J.F., and Pruvost, J. (2010). Experimental and theoretical assessment of maximum productivities for the microalgae chlamydomonas reinhardtii in two different geometries of photobioreactors. Biotechnology progress, 26(2), 431-440.

Tebbani, S., Lopes, F., Filali, R., Dumur, D., and Pareau, D. (2013). Nonlinear predictive control for maximization of co2 bio-fixation by microalgae in a photobioreactor. Bioprocess and biosystems engineering, 1-15.

Weissing, F. and Huisman, J. (1994). Growth and competition in a light gradient. Journal of theoretical biology, 168, 323-336.

Wijffels, R. and Barbosa, M. (2010). An Outlook on Microalgal Biofuels. Science, 329(5993), 796-799. 